\title{
Some notes on the gh-lifts of affine connections
}

\author{
Rabia Cakan Akpınar
}

Received: 05.11.2019 / Revised: 05.04.2020 / Accepted: 08.05.2020

\begin{abstract}
Let $M$ be a differentiable manifold with dimension $n$ and $T^{*} M$ be its cotangent bundle. In this paper, we determine the gh - lift of the affine connection via the musical isomorphism on the cotangent bundle $T^{*} M$. We obtain the torsion tensor, curvature tensor and geodesic curve of the gh-lift of LeviCivita connection.
\end{abstract}

Keywords. Horizontal lift, connection, curvature tensor, musical isomorphism, geodesic.

Mathematics Subject Classification (2010): 55R10, 53C05

\section{Introduction}

The connections were studied from an infinitesimal perspective in Riemannian geometry. Several authors investigated the lifts of connections on the different type bundle. Pogoda [8] defined the horizontal lift of basic connections of order $r$ to a transverse naturel vector bundle and studied its properties. Salimov and Fattayev [9] determined the horizontal lift and complete lifts of the linear connection from a smooth manifold to its coframe bundle. Several authors investigated the lifts of connections on the cotangent bundle $T^{*} M$. Yano and Patterson [10] applied the horizontal lifts which was defined by them to the symmetric connections on the cotangent bundle $T^{*} M$. Kures [6] determined all natural operators transforming classical torsion-free linear connections on a manifold $M$ into classical linear connections on the cotangent bundle $T^{*} M$. Also the Riemannian manifolds and the cotangent bundles have been studied by many authors $[3,7,4,5]$. In this paper, calculating the coefficients of $g h-$ lift $G H \stackrel{*}{\nabla}$ of the affine connection via the musical isomorphism we have determined the $g h-$ lift $G H \stackrel{*}{\nabla}$ of the affine connection $\nabla$. After using the coefficients of the $g h$-lift $G H \stackrel{*}{\nabla}$ we have determined the torsion tensor and the curvature tensor of the $g h$-lift $G H \stackrel{*}{\nabla}$ of Levi-Civita connection. Finally we have investigated properties of the geodesic of the $g h-$ lift $G H \stackrel{*}{\nabla}$ to the cotangent bundle $T^{*} M$. 


\section{Preliminaries}

Let $M$ be a pseudo-Riemannian manifold with $n$ dimension. The tangent bundle on $M$ is denoted by $T M=\cup_{x \in M} T_{x} M$. The local coordinates on $T M$ are $\left(x^{i}, x^{\bar{i}}\right)=\left(x^{i}, y^{i}\right)$ where $\left(x^{i}\right)$ are local coordinates on $M$ and $\left(y^{i}\right)$ are vector space coordinates according to the basis $\partial / \partial x^{i}$, i.e. $y_{x}=y^{i} \frac{\partial}{\partial x^{i}} \in T_{x} M$. The cotangent bundle on $M$ is denoted by $T^{*} M=\cup_{x \in M} T_{x}^{*} M$. The local coordinates on $T^{*} M$ are $\left(x^{i}, \widetilde{x}^{\bar{i}}\right)=\left(x^{i}, p_{i}\right)$ where $\left(x^{i}\right)$ are local coordinates on $M$ and $p_{i}$ are vector space coordinates according to the basis $d x^{i}$, i.e. $p_{x}=p_{i} d x^{i} \in T_{x}^{*} M$. We denote by $\Im_{s}^{r}(M)$ the set of all tensor fields of type $(r, s)$ on $M$. Throughout this paper we assume the manifolds, tensor fields and connections to be diferentiable of class $C^{\infty}$. We use the ranges of the index $i$ being $\{1, \ldots, n\}$ and the index $\bar{i}$ being $\{n+1, \ldots, 2 n\}$.

Let $g$ be a pseudo Riemannian metric. $g^{\sharp}: T^{*} M \rightarrow T M$ is the musical isomorphism associated with $g$ pseudo Riemannian metric with inverse given by $g^{b}: T M \rightarrow T^{*} M$.

The musical isomorphism $g^{\sharp}$ described by

$$
g^{\sharp}: \tilde{x}^{M}=\left(x^{m}, \tilde{x}^{\bar{m}}\right)=\left(x^{m}, p_{m}\right) \rightarrow x^{J}=\left(x^{j}, x^{\bar{j}}\right)=\left(\delta_{m}^{j} x^{m}, y^{j}=g^{j m} p_{m}\right) .
$$

In music notation, the sharp symbol $\sharp$ increase a note by a half step. And similar to this musical notation, the musical isomorphism $g^{\sharp}$ increase the indice of the vector space coordinate.

The musical isomorphism $g^{b}$ is described by

$$
g^{b}: x^{I}=\left(x^{i}, x^{\bar{i}}\right)=\left(x^{i}, y^{i}\right) \rightarrow \tilde{x}^{K}=\left(x^{k}, \tilde{x}^{\bar{k}}\right)=\left(\delta_{i}^{k} x^{i}, p_{k}=g_{k i} y^{i}\right) .
$$

In another music notation, the flat symbol $b$ lowers a note by a half step. Similar to this musical notation, the musical isomorphism $g^{b}$ low the indice of the vector space coordinate.

The Jacobian matrice of $g^{b}$ is obtained by

$$
\left(g_{*}^{b}\right)=\left(\widetilde{A}_{J}^{M}\right)=\left(\frac{\partial \tilde{x}^{M}}{\partial x^{J}}\right)=\left(\begin{array}{cc}
\delta_{j}^{m} & 0 \\
y^{s} \partial_{j} g_{m s} & g_{m j}
\end{array}\right)
$$

and the Jacobian matrice of $g^{\sharp}$ is obtained by

$$
\left(g_{*}^{\sharp}\right)=\left(A_{M}^{J}\right)=\left(\frac{\partial x^{J}}{\partial \tilde{x}^{M}}\right)=\left(\begin{array}{cc}
\delta_{m}^{j} & 0 \\
p_{s} \partial_{m} g^{j s} & g^{j m}
\end{array}\right),
$$

where $\delta$ is the Kronecker delta [1].

Yano and Patterson [10] defined the horizontal lift and applied this notion to connection in $M$. The $g h$-lift is newly defined in [2]. We obtain gh-lift ${ }^{G H} \stackrel{*}{\nabla}$ of the affine connection $\nabla$ by transferring the horizontal lift of affine connection $\nabla$ from tangent bundle to cotangent bundle via the musical isomorphism. The problems of transferring the lifts to the cotangent bundle were studied in $[1,2]$.

\section{The $g h-$ lift of affine connection}

Let $\nabla$ be an affine connection on $M$ manifold. The horizontal lift ${ }^{H} \nabla$ of the affine connection $\nabla$ to the tangent bundle $T M$ is formed with equation

$$
{ }^{H} \nabla={ }^{C} \nabla-{ }^{V} R
$$


where ${ }^{V} R$ is vertical lift of curvature tensor of $\nabla$ and ${ }^{C} \nabla$ is complete lift of affine connection to the tangent bundle $T M$. And also the horizontal lift ${ }^{H} \nabla$ of the affine connection $\nabla$ provides the conditions

$$
\begin{aligned}
{ }^{H} \nabla_{V_{X}}{ }^{V} Y & =0, & { }^{H} \nabla_{V_{X}}{ }^{H} Y=0, \\
{ }^{H} \nabla_{H_{X}}{ }^{V} Y & ={ }^{V}\left(\nabla_{X} Y\right), & { }^{H} \nabla_{H_{X}}{ }^{H} Y={ }^{H}\left(\nabla_{X} Y\right)
\end{aligned}
$$

for any $X, Y \in \Im_{0}^{1}(M)$.

Let $\Gamma_{a b}^{c}$ be coefficient of $\nabla$ according to the local coordinates $\left(x^{h}\right)$ on $M$. Let ${ }^{H} \Gamma_{A B}^{C}$ be coefficient of ${ }^{H} \nabla$ according to the induced coordinates $\left(x^{h}, y^{h}\right)$ to the tangent bundle $T M$. The non-zero coefficients ${ }^{H} \Gamma_{A B}^{C}$ of the horizontal lift ${ }^{H} \nabla$ to the tangent bundle $T M$ are given by

$$
{ }^{H} \Gamma_{a b}^{c}=\Gamma_{a b}^{c},{ }^{H} \Gamma_{a b}^{\bar{c}}=\Gamma_{a b}^{c},{ }^{H} \Gamma_{\bar{a} b}^{\bar{c}}=\Gamma_{a b}^{c},{ }^{H} \Gamma_{a b}^{\bar{c}}=y^{s} \partial_{s} \Gamma_{a b}^{c}-y^{s} R_{s a b}^{c}
$$

where $R_{s a b}^{c}$ is the curvature tensor of $\nabla$.

The horizontal lift ${ }^{H} \stackrel{*}{\nabla}$ of the affine connection $\nabla$ to the cotangent bundle $T^{*} M$ is formed with equation

$$
{ }^{H} \stackrel{*}{\nabla}={ }^{C} \stackrel{*}{\nabla}-V_{R}^{*}
$$

where $\nabla$ is symmetric. ${ }^{V} \stackrel{*}{R}$ is vertical lift of curvature tensor of $\nabla$ and ${ }^{C} \stackrel{*}{\nabla}$ is complete lift of $\nabla$ to the cotangent bundle $T^{*} M$. Let ${ }^{H}{ }_{\Gamma}^{*}{ }_{A B}^{C}$ be coefficient of ${ }^{H} \stackrel{*}{\nabla}_{\text {according to the induced }}$ coordinates $\left(x^{h}, p_{h}\right)$ to the cotangent bundle $T^{*} M$. The non-zero coefficients ${ }^{H}{ }_{\Gamma}^{*}{ }_{A B}^{C}$ of the horizontal lift ${ }^{*} \stackrel{*}{\nabla}$ are given by

$$
{ }^{H} \stackrel{*}{\Gamma}_{a b}^{c}=\Gamma_{a b}^{c},{ }^{H} \stackrel{*}{\Gamma}_{a \bar{b}}^{\bar{c}}=-\Gamma_{a c}^{b},{ }^{H} \stackrel{*}{\Gamma}_{\bar{a} b}^{\bar{c}}=-\Gamma_{c b}^{a},{ }^{H} \stackrel{*}{\Gamma}_{a b}^{\bar{c}}=p_{s}\left(-\partial_{a} \Gamma_{b c}^{s}+\Gamma_{k c}^{s} \Gamma_{a b}^{k}+\Gamma_{b t}^{s} \Gamma_{a c}^{t}\right)
$$

according to the induced coordinates $\left(x^{h}, p_{h}\right)$ to the cotangent bundle $T^{*} M$ [11].

We obtain the $\frac{\partial^{2} \widetilde{x}^{K}}{\partial x^{A} \partial x^{B}}$ that have components

$$
\begin{aligned}
& \frac{\partial^{2} \widetilde{x}^{k}}{\partial x^{a} \partial x^{b}}=0, \frac{\partial^{2} \widetilde{x}^{k}}{\partial x^{a} \partial x^{\bar{b}}}=0, \quad \frac{\partial^{2} \widetilde{x}^{k}}{\partial x^{\bar{a}} \partial x^{b}}=0, \quad \frac{\partial^{2} \widetilde{x}^{k}}{\partial x^{\bar{a}} \partial x^{\bar{b}}}=0 \\
& \frac{\partial^{2} \widetilde{x}^{\bar{k}}}{\partial x^{a} \partial x^{b}}=\frac{p_{s} \partial^{2} g^{k s}}{\partial x^{a} \partial x^{b}}, \quad \frac{\partial^{2} \widetilde{x}^{\bar{k}}}{\partial x^{a} \partial x^{\bar{b}}}=\partial_{a} g^{k b}, \quad \frac{\partial^{2} \widetilde{x}^{\bar{k}}}{\partial x^{\bar{a}} \partial x^{b}}=\partial_{b} g^{k a}, \quad \frac{\partial^{2} \widetilde{x}^{\bar{k}}}{\partial x^{\bar{a}} \partial x^{\bar{b}}}=0 .
\end{aligned}
$$

And using $(2.3),(2.4),(3.3)$ and $(3.6)$, the coefficients $G H \stackrel{*}{\Gamma}{ }_{A B}^{C}$ of $G H \stackrel{*}{\nabla}$ are obtained from equation

$$
g_{*}^{b H} \Gamma_{A B}^{C}=\left({ }^{G H} \stackrel{*}{\Gamma}_{A B}^{C}\right)=\left(\frac{\partial \tilde{x}^{M}}{\partial x^{A}} \frac{\partial \tilde{x}^{S}}{\partial x^{B}} \frac{\partial x^{C}}{\partial \tilde{x}^{K}}{ }^{H} \Gamma_{M S}^{K}+\frac{\partial x^{C}}{\partial \tilde{x}^{K}} \frac{\partial^{2} \tilde{x}^{K}}{\partial x^{A} \partial x^{B}}\right) .
$$

The non-zero coefficients $G H_{\Gamma}^{*}{ }_{A B}^{C}$ of $G H \stackrel{*}{\nabla}$ are obtained as follows where $A, B, \ldots=$ $1, \ldots, 2 n$ : 


$$
\begin{aligned}
& G H_{\Gamma}^{*}{ }_{a b}^{c}=\delta_{a}^{m} \delta_{b}^{s} \delta_{k}^{c} \Gamma_{m s}^{k}=\Gamma_{a b}^{c} \\
& { }^{G H} \stackrel{\Gamma}{\Gamma}_{a \bar{b}}^{\bar{c}}=\delta_{a}^{m} g^{s b} g_{c k} \Gamma_{m s}^{k}+g_{c k} \partial_{a} g^{k b} \\
& =\Gamma_{a c}^{b}-g^{k b} \partial_{a} g_{c k} \\
& =-\Gamma_{a c}^{b}+g^{b k} \partial_{c} g_{a k}-g^{b k} \partial_{k} g_{a c} \\
& =-\Gamma_{a c}^{b}+g^{b k}\left(\nabla_{c} g_{a k}+\Gamma_{c a}^{t} g_{t k}+\Gamma_{c k}^{t} g_{a t}\right)-g^{b k}\left(\nabla_{k} g_{a c}+\Gamma_{k a}^{t} g_{t c}+\Gamma_{k c}^{t} g_{t a}\right) \\
& =-\Gamma_{a c}^{b}+g^{b k} \nabla_{c} g_{a k}+g^{b k} \Gamma_{c a}^{t} g_{t k}-g^{b k} \nabla_{k} g_{a c}-g^{b k} \Gamma_{k a}^{t} g_{t c} \\
& =-\Gamma_{a c}^{b} \\
& G H \stackrel{*}{\Gamma} \frac{\bar{c}}{a} b=g^{m a} \delta_{b}^{s} g_{c k} \Gamma_{m s}^{k}+g_{c k} \partial_{b} g^{k a} \\
& =\Gamma_{c b}^{a}-g^{k a} \partial_{b} g_{c k} \\
& =-\Gamma_{c b}^{a}+g^{a k} \partial_{c} g_{b k}-g^{a k} \partial_{k} g_{c b} \\
& =-\Gamma_{c b}^{a}+g^{a k}\left(\nabla_{c} g_{b k}+\Gamma_{c k}^{t} g_{b t}+\Gamma_{c b}^{t} g_{t k}\right)-g^{a k}\left(\nabla_{k} g_{c b}+\Gamma_{k c}^{t} g_{t b}+\Gamma_{k b}^{t} g_{c t}\right) \\
& \begin{array}{l}
=-\Gamma_{c b}^{a}+g^{a k} \nabla_{c} g_{b k}+g^{a k} \Gamma_{c b}^{t} g_{t k}-g^{a k} \nabla_{k} g_{c b}-g^{a k} \Gamma_{k b}^{t} g_{c t} \\
=-\Gamma_{c b}^{a}
\end{array} \\
& G H \stackrel{F}{\Gamma}_{a b}^{\bar{c}}=\delta_{a}^{m} \delta_{b}^{s} y^{t} \partial_{k} g_{t c} \Gamma_{m s}^{k}+\delta_{a}^{m} \delta_{b}^{s} g_{c k}\left(y^{t} \partial_{t} \Gamma_{m s}^{k}-y^{t} R_{t m s}^{k}\right)+p_{t} \partial_{a} g^{m t} \delta_{b}^{s} g_{c k} \Gamma_{m s}^{k} \\
& +\delta_{a}^{m} p_{t} \partial_{b} g^{s t} g_{c k} \Gamma_{m s}^{k}+g_{c k} p_{t} \partial_{a b}^{2} g^{t k} \\
& =y^{t}\left(\partial_{k} g_{t c}\right) \Gamma_{a b}^{k}+g_{c k} y^{t} \partial_{t} \Gamma_{a b}^{k}-g_{c k} y^{t} R_{t a b}^{k}+p_{t} \partial_{a} g^{m t} g_{c k} \Gamma_{m b}^{k}+p_{t} \partial_{b} g^{s t} g_{c k} \Gamma_{a s}^{k} \\
& +g_{c k} p_{t} \partial_{a b}^{2} g^{t h} \\
& =y^{t}\left(\partial_{k} g_{t c}\right) \Gamma_{a b}^{k}+g_{c k} y^{t} \partial_{t} \Gamma_{a b}^{k}-g_{c k} y^{t} R_{t a b}^{k}+p_{t} \partial_{a} g^{m t} g_{c k} \Gamma_{m b}^{k}+p_{t} \partial_{b} g^{s t} g_{c k} \Gamma_{a s}^{k} \\
& -p_{t}\left(\partial_{a} \Gamma_{b c}^{t}\right)-g_{c k} p_{t} \Gamma_{b s}^{t} \partial_{a} g^{s k}-g_{c k} p_{t}\left(\partial_{a} \Gamma_{b s}^{k}\right) g^{t s}-g_{c k} p_{t} \Gamma_{b s}^{k} \partial_{a} g^{t s} \\
& =-p_{t}\left(\partial_{a} \Gamma_{b c}^{t}\right)+y^{t}\left(\partial_{k} g_{t c}\right) \Gamma_{a b}^{k}+g_{c k} y^{t} \partial_{t} \Gamma_{a b}^{k}-g_{c k} y^{t} R_{t a b}^{k}+p_{t}\left(\partial_{b} g^{s t}\right) g_{c k} \Gamma_{a s}^{k} \\
& -g_{c k} p_{t} \Gamma_{b s}^{t} \partial_{c} g^{s k}-g_{c k} p_{t}\left(\partial_{a} \Gamma_{b s}^{k}\right) g^{t s} \\
& =-p_{t}\left(\partial_{a} \Gamma_{b c}^{t}\right)+y^{t}\left(\partial_{k} g_{t c}\right) \Gamma_{a b}^{k}+g_{c k} y^{t} \partial_{t} \Gamma_{a b}^{k}-g_{c k} y^{t} R_{t a b}^{k}-g_{c k} p_{t}\left(\partial_{a} \Gamma_{b s}^{k}\right) g^{t s} \\
& +p_{t}\left(\partial_{b} g^{s t}\right) g_{c k} \Gamma_{a s}^{k}-g_{c k} p_{t} \Gamma_{b s}^{t} \partial_{a} g^{s k} \\
& =-p_{t}\left(\partial_{a} \Gamma_{b c}^{t}\right)+y^{t}\left(\partial_{k} g_{t c}\right) \Gamma_{a b}^{k}+g_{c k}\left(g^{t s} p_{s} \partial_{t} \Gamma_{a b}^{k}-p_{t} g^{t s} \partial_{a} \Gamma_{b s}^{k}-y^{t} R_{t a b}^{k}\right) \\
& +p_{t}\left(-\Gamma_{b m}^{s} g^{m t}-\Gamma_{b m}^{t} g^{s m}\right) g_{c k} \Gamma_{a s}^{k}-g_{c k} p_{t} \Gamma_{b s}^{t}\left(-\Gamma_{a m}^{s} g^{m k}-\Gamma_{a m}^{k} g^{m s}\right) \\
& =-p_{t}\left(\partial_{a} \Gamma_{b c}^{t}\right)+p_{s} \Gamma_{c k}^{s} \Gamma_{a b}^{k}+g^{t s} g_{m c} p_{s} \Gamma_{k t}^{m} \Gamma_{a b}^{k}+g_{c k} g^{t s} p_{s}\left(\partial_{t} \Gamma_{a b}^{k}-\partial_{a} \Gamma_{b t}^{k}\right) \\
& -g_{c k} g^{t s} p_{s} R_{t a b}^{k}-g_{c k} p_{s}\left(\Gamma_{b m}^{t} \Gamma_{a t}^{k} g^{m s}+\Gamma_{b m}^{s} \Gamma_{a t}^{k} g^{t m}\right) \\
& +g_{h k} p_{s}\left(\Gamma_{b t}^{s} \Gamma_{a m}^{t} g^{m k}+\Gamma_{b t}^{s} \Gamma_{a m}^{k} g^{m t}\right) \\
& =-p_{t}\left(\partial_{a} \Gamma_{b c}^{t}\right)+p_{s} \Gamma_{c k}^{s} \Gamma_{a b}^{k}+p_{s} \Gamma_{k c}^{s} \Gamma_{a b}^{k}+p_{s} \partial_{c} \Gamma_{a b}^{s}-p_{s} \partial_{a} \Gamma_{b c}^{s}-p_{s} \partial_{c} \Gamma_{a b}^{s} \\
& +p_{s} \partial_{a} \Gamma_{c b}^{s}-p_{s} \Gamma_{c m}^{s} \Gamma_{a b}^{m}+p_{s} \Gamma_{a m}^{s} \Gamma_{c b}^{m}-p_{s} \Gamma_{a t}^{s} \Gamma_{b c}^{t}-p_{s} \Gamma_{b m}^{s} \Gamma_{a c}^{m}+p_{s} \Gamma_{b t}^{s} \Gamma_{a c}^{t} \\
& +p_{s} \Gamma_{b t}^{s} \Gamma_{a c}^{t} \\
& =-p_{s} \partial_{a} \Gamma_{b c}^{s}+p_{s} \Gamma_{k c}^{s} \Gamma_{a b}^{k}+p_{s} \Gamma_{b t}^{s} \Gamma_{a c}^{t} \\
& =p_{s}\left(-\partial_{a} \Gamma_{b c}^{s}+\Gamma_{k c}^{s} \Gamma_{a b}^{k}+\Gamma_{b t}^{s} \Gamma_{a c}^{t}\right)
\end{aligned}
$$

After these results we get the following theorem

Theorem 3.1 Let $(M, g)$ be a pseudo Riemannian manifold with dimension $n$. Let $\nabla$ be an affine connection on manifold $M$ and ${ }^{H} \nabla$ be the horizontal lift of $\nabla$ to the tangent bundle $T M$. Then the differential of ${ }^{H} \nabla$ by $g^{b}$, i.e., a gh-lift ${ }^{G H} \stackrel{*}{\nabla}$ to the cotangent bundle $T^{*} M$ coincides with the horizontal lift ${ }^{H} \stackrel{*}{\nabla}$ to the cotangent bundle $T^{*} M$ if $\nabla$ is a Levi-Civita connection. 


\section{The Torsion and Curvature Tensors of $g h$ - lift of Levi-Civita Connection}

Let $\widetilde{T}$ be torsion tensor of horizontal lift of the symmetric affine connection $\nabla$ on $M$ to the cotangent bundle $T^{*} M$. The torsion tensor $\widetilde{T}$ of ${ }^{H} \stackrel{*}{\nabla}$ is determined by

$$
\widetilde{T}\left({ }^{V} \theta,{ }^{V} \omega\right)=0, \quad \widetilde{T}\left({ }^{H} X,{ }^{V} \omega\right)=0, \quad \widetilde{T}\left({ }^{H} X,{ }^{H} Y\right)=-\gamma R(X, Y)
$$

where $R$ is the curvature tensor of $\nabla \cdot{ }^{H} X,{ }^{H} Y$ are horizontal lift of $X, Y \in \Im_{0}^{1}(M)$ and ${ }^{V} \theta, V_{\omega}$ are vertical lift of $\theta, \omega \in \Im_{1}^{0}(M)$ to the cotangent bundle $T^{*} M$. The non zero component $\widetilde{T}_{A B}^{C}$ of $\widetilde{T}$ is given

$$
\widetilde{T}_{a b}^{\bar{c}}=-p_{t} R_{a b c}^{t}
$$

according to the induced coordinates $\left(x^{h}, p_{h}\right)$ to the cotangent bundle $T^{*} M$ [11].

Let $\widehat{T}$ be torsion tensor of the gh-lift $G H \stackrel{*}{\nabla}$ of the Levi-Civita connection on $M$ to the cotangent bundle $T^{*} M$. Using the coefficient $G H \Gamma^{*}{ }_{A B}^{C}$ of ${ }^{G H}{ }^{*}$ to the cotangent bundle $T^{*} M$ the components $\widehat{T}_{A B}^{C}$ of $\widehat{T}$ are obtained with the equation

$$
\widehat{T}_{A B}^{C}=G H{ }_{\Gamma}^{*}{ }_{A B}^{C}-G H{ }_{\Gamma}^{*}{ }_{B A}^{C}
$$

according to the induced coordinates $\left(x^{h}, p_{h}\right)$ to the cotangent bundle $T^{*} M$. We have

$$
\begin{array}{llll}
\widehat{T}_{a b}^{c}=0, & \widehat{T}_{\bar{a} b}^{c}=0, & \widehat{T}_{a \bar{b}}^{c}=0, & \widehat{T}_{a b}^{\bar{c}}=-p_{t} R_{a b c}^{t} \\
\widehat{T}_{\bar{a} b}^{\bar{c}}=0, & \widehat{T}_{a \bar{b}}^{\bar{c}}=0, & \widehat{T}_{\bar{a} \bar{b}}^{c}=0, & \widehat{T}_{\bar{a} \bar{b}}^{\bar{c}}=0 .
\end{array}
$$

After we have

Corollary 4.1 The torsion tensor $\widehat{T}$ of the gh-lift ${ }^{G H} \stackrel{*}{\nabla}$ of Levi-Civita connection coincides with the torsion tensor $\widetilde{T}$ of horizontal lift ${ }^{H} \stackrel{*}{\nabla}$ of the symmetric affine connection.

Let $\widetilde{R}$ be curvature tensor of horizontal lift of the symmetric affine connection $\nabla$ on $M$ to the cotangent bundle $T^{*} M$. For any $X, Y, Z \in \Im_{0}^{1}(M)$ and $\omega, \theta, \psi \in \Im_{1}^{0}(M)$, the curvature tensor $\widetilde{R}$ of ${ }^{H} \stackrel{*}{\nabla}$ provides the conditions

$$
\begin{aligned}
\widetilde{R}\left({ }^{V} \theta,{ }^{V} \omega\right) & =0, \quad \widetilde{R}\left({ }^{H} X,{ }^{V} \omega\right)=0 \\
\widetilde{R}\left({ }^{H} X,{ }^{H} Y\right){ }^{V} \psi & =-{ }^{V}(\psi \circ R(X, Y)) \\
\widetilde{R}\left({ }^{H} X,{ }^{H} Y\right){ }^{H} Z & ={ }^{H}(R(X, Y) Z)
\end{aligned}
$$

where $R$ is the curvature tensor of $\nabla$. The non zero components $\widetilde{R}_{A B D}^{C}$ of $\widetilde{R}$ are given

$$
\begin{aligned}
\widetilde{R}_{a b d}^{c} & =R_{a b d}^{c} \\
\widetilde{R}_{a b d}^{\bar{c}} & =p_{s}\left(\Gamma_{c t}^{s} R_{a b d}^{t}+\Gamma_{d t}^{s} R_{a b c}^{t}\right) \\
\widetilde{R}_{a b \bar{d}}^{\bar{c}} & =-R_{a b c}^{d}
\end{aligned}
$$

according to the induced coordinates $\left(x^{h}, p_{h}\right)$ to the cotangent bundle $T^{*} M$. 
Let $\widehat{R}$ be curvature tensor of the gh-lift ${ }^{G H} \stackrel{*}{\nabla}$ of Levi-Civita connection on $M$ to the cotangent bundle $T^{*} M$. Using the coefficient $G H{ }_{\Gamma}^{*}{ }_{A B}^{C}$ of $G H \stackrel{*}{\nabla}$ to the cotangent bundle $T^{*} M$ the components $\widehat{R}_{A B D}^{C}$ of $\widehat{R}$ are obtained with the equation

$$
\widehat{R}_{A B D}^{C}=\partial_{A} G H{ }_{\Gamma}^{*}{ }_{B D}^{C}-\partial_{B} G H{ }_{\Gamma}^{*}{ }_{A D}^{C}+G H_{\Gamma}^{*}{ }_{A T}^{C} G H_{\Gamma}^{*}{ }_{B D}^{T}-G H{ }_{\Gamma}^{*}{ }_{B T}^{C} G H{ }_{\Gamma}^{*}{ }_{A D}^{T}
$$

according to the induced coordinates $\left(x^{h}, p_{h}\right)$. We have

$$
\begin{aligned}
& \widehat{R}_{a b d}^{c}=R_{a b d}^{c} \\
& \widehat{R}_{a b d}^{c}=p_{s}\left(\Gamma_{c t}^{s} R_{a b d}^{t}+\Gamma_{d t}^{s} R_{a b c}^{t}\right) \\
& \widehat{R}_{a b \bar{d}}^{c}=-R_{a b c}^{d} .
\end{aligned}
$$

After we have

Corollary 4.2 The curvature tensor $\widehat{R}$ of the gh-lift ${ }^{G H} \stackrel{*}{\nabla}$ of Levi-Civita connection coincides with the curvature tensor $\widetilde{R}$ of horizontal lift ${ }^{H} \stackrel{*}{\nabla}$ of the symmetric affine connection.

\section{Geodesics of $g h$-lift of Levi-Civita Connection}

Let $\widetilde{C}$ be a geodesic curve of the gh-lift ${ }^{G H} \stackrel{*}{\nabla}$ of Levi-Civita connection on $M$ to the cotangent bundle $T^{*} M$. The geodesic $\widetilde{C}$ is determined with the equations

$$
\frac{d^{2} x^{C}}{d t^{2}}+{ }^{G H} \stackrel{\Gamma}{C}_{A B}^{C} \frac{d x^{A}}{d t} \frac{d x^{B}}{d t}=0
$$

according to the induced coordinates $\left(x^{c}, x^{\bar{c}}\right)=\left(x^{c}, p_{c}\right)$.

Using the coefficient of gh-lift $G H \stackrel{*}{\nabla}$ we obtain following equations from (5.1):

$$
\begin{aligned}
& \frac{d^{2} x^{c}}{d t^{2}}+G H \stackrel{*}{\Gamma}_{a b}^{c} \frac{d x^{a}}{d t} \frac{d x^{b}}{d t}+G H \stackrel{*}{\Gamma}_{\bar{a} b} \frac{d x^{\bar{a}}}{d t} \frac{d x^{b}}{d t}+G H{ }^{* c} \underset{\Gamma_{a b}}{ } \frac{d x^{a}}{d t} \frac{d x^{\bar{b}}}{d t}+G H \stackrel{*}{\Gamma}_{\bar{a}}^{c} \frac{d x^{\bar{a}}}{d t} \frac{d x^{\bar{b}}}{d t}=0 \\
& \frac{d^{2} x^{c}}{d t^{2}}+\Gamma_{a b}^{c} \frac{d x^{a}}{d t} \frac{d x^{b}}{d t}=0, \\
& \frac{d^{2} x^{\bar{c}}}{d t^{2}}+G H \stackrel{*}{\Gamma}_{a b} \frac{d x^{a}}{d t} \frac{d x^{b}}{d t}+G H \stackrel{* \bar{c}}{\Gamma_{\bar{a}}} \frac{d x^{\bar{a}}}{d t} \frac{d x^{b}}{d t}+G H \stackrel{* \bar{c}}{\Gamma_{a}} \frac{d x^{a}}{d t} \frac{d x^{\bar{b}}}{d t}+G H \stackrel{* \bar{c}}{\Gamma_{\bar{a}} \bar{b} \frac{d x^{\bar{a}}}{d t}} \frac{d x^{\bar{b}}}{d t}=0 \\
& \frac{d^{2} p_{c}}{d t^{2}}+p_{s}\left(-\partial_{a} \Gamma_{b c}^{s}+\Gamma_{c m}^{s} \Gamma_{a b}^{m}+\Gamma_{b m}^{s} \Gamma_{c a}^{m}\right) \frac{d x^{a}}{d t} \frac{d x^{b}}{d t}-\Gamma_{c b}^{a} \frac{d p_{a}}{d t} \frac{d x^{b}}{d t}-\Gamma_{a c}^{b} \frac{d x^{a}}{d t} \frac{d p_{b}}{d t}=0 \\
& \frac{d}{d t}\left(\frac{d p_{c}}{d t}-\Gamma_{c b}^{a} p_{a} \frac{d x^{b}}{d t}\right)-\Gamma_{a c}^{m}\left(\frac{d p_{m}}{d t}-\Gamma_{b m}^{s} p_{s} \frac{d x^{b}}{d t}\right) \frac{d x^{a}}{d t}=0 \\
& \frac{d}{d t}\left(\frac{\delta p_{c}}{d t}\right)-\Gamma_{a c}^{m}\left(\frac{\delta p_{m}}{d t}\right) \frac{d x^{a}}{d t}=0 \\
& \begin{array}{c}
\frac{\delta}{d t}\left(\frac{\delta p_{c}}{d t}\right)=0 \\
\frac{\delta^{2} p_{c}}{d t^{2}}=0
\end{array}
\end{aligned}
$$

where $\frac{\delta p_{c}}{d t}=\frac{d p_{c}}{d t}-\Gamma_{c b}^{a} p_{a} \frac{d x^{b}}{d t}$. After expressions (5.2) and (5.3) we have 
Theorem 5.1 Let $\widetilde{C}$ be a geodesic according to the gh-lift ${ }^{G H} \stackrel{*}{\nabla}$ of Levi-Civita connecion on $M$ to the cotangent bundle $T^{*} M$. The geodesic $\widetilde{C}$ has the eqations

$$
\begin{gathered}
\frac{d^{2} x^{c}}{d t^{2}}+\Gamma_{a b}^{c} \frac{d x^{a}}{d t} \frac{d x^{b}}{d t}=0, \\
\frac{\delta^{2} p_{c}}{d t^{2}}=0
\end{gathered}
$$

according to the induced coordinates $\left(x^{c}, p_{c}\right)$ to $T^{*} M$.

Theorem 5.2 The curve $\widetilde{C}$ on the cotangent bundle $T^{*} M$ is a geodesic according to the $g h$ lift ${ }^{G H} \stackrel{*}{\nabla}$ of Levi-Civita connecion on $M$ if the projection $C=\pi(\widetilde{C})$ on $M$ is a geodesic according to the $\nabla$ on $M$ and the second covariant differentiation of $p_{c}=p_{c}(t)$ along $C$ vanishes where $\pi: T^{*} M \rightarrow M$ is the naturel projection.

\section{References}

1. Cakan, R., Akbulut, K., Salimov, A.: Musical isomorphisms and problems of lifts, Chin. Ann. Math. 37 B(3), 323-330 (2016).

2. Cakan, R.: On gh-lifts of some tensor fields, C. R. Acad. Bulg. Sci. 71 (3), 317-324 (2018).

3. Cayır, H.: Derivatives with respect to lifts of the riemannian metric of the format ${ }^{f} \tilde{G}={ }^{S} g_{f}+{ }^{H} g$ on TM over a riemannian manifold $(M, g)$, Punjab University Journal of Mathematics. 51 (1), 1-8 (2019).

4. Druta Romaniuc, S.L.: Natural diagonal riemannian almost product and parahermitian cotangent bundles, Czechoslovak Math. J. 62 (4), 937-949 (2012).

5. Kurek, J., Mikulski, W.M.: The natural operators lifting connections to tensor powers of the cotangent bundle, Miskolc Math. Notes. 14, 517-524 (2013).

6. Kures, M.: Naturel lifts of classical linear connections to the cotangent bundle, Suppl. Rend. Circolo Mat. Palermo II. 43, 181-187 (1996).

7. Mok, K.P.: Metrics and connections on the cotangent bundle, Kodai Math. Sem. Rep. 28 (2-3), 226-238 (1977).

8. Pogoda, Z.: Horizontal lifts and foliations. Suppl. Rend. Circolo Mat. Palermo II. 21, 279-289 (1989).

9. Salimov, A., Fattayev H.D.: Connections on the coframe bundle, Int. Electron. J. Geom. 12 (1), 93-101 (2019).

10. Yano, K., Patterson, E.M.: Horizontal lifts from a manifold to its cotangent bundle, J. Math. Soc. Japan. 19, 185-198 (1967).

11. Yano, K., Ishihara, S.: Tangent and Cotangent Bundles, Marcel Dekker (1973). 